\title{
Laboratory-based micro-X-ray fluorescence setup using a von Hamos crystal spectrometer and a focused beam X-ray tube
}

\author{
Y. Kayser, ${ }^{1,2, a)}$ W. Błachucki, ${ }^{1}$ J.-Cl. Dousse, ${ }^{1}$ J. Hoszowska, ${ }^{1}$ M. Neff, ${ }^{3}$ and V. Romano ${ }^{3}$ \\ ${ }^{1}$ Department of Physics, University of Fribourg, 1700 Fribourg, Switzerland \\ ${ }^{2}$ Paul Scherrer Institut, 5232 Villigen-PSI, Switzerland \\ ${ }^{3}$ Institute of Applied Physics, University of Bern, 3012 Bern, Switzerland
}

(Received 24 February 2014; accepted 12 March 2014; published online 1 April 2014)

\begin{abstract}
The high-resolution von Hamos bent crystal spectrometer of the University of Fribourg was upgraded with a focused X-ray beam source with the aim of performing micro-sized X-ray fluorescence (XRF) measurements in the laboratory. The focused X-ray beam source integrates a collimating optics mounted on a low-power micro-spot X-ray tube and a focusing polycapillary half-lens placed in front of the sample. The performances of the setup were probed in terms of spatial and energy resolution. In particular, the fluorescence intensity and energy resolution of the von Hamos spectrometer equipped with the novel micro-focused X-ray source and a standard high-power water-cooled X-ray tube were compared. The XRF analysis capability of the new setup was assessed by measuring the dopant distribution within the core of Er-doped $\mathrm{SiO}_{2}$ optical fibers. () 2014 AIP Publishing LLC. [http://dx.doi.org/10.1063/1.4869340]
\end{abstract}

\section{INTRODUCTION}

Micro-X-ray fluorescence ( $\mu$-XRF) analysis ${ }^{1}$ is applied in environmental sciences, ${ }^{2,3}$ geological applications, ${ }^{4,5}$ cultural heritage studies, ${ }^{6}$ archeology, ${ }^{7}$ and in the biomedical, ${ }^{8,9}$ chemical, ${ }^{10,11}$ and forensic ${ }^{12}$ domains. Usually the related experiments are realized at advanced research facilities, i.e., third generation synchrotron sources, where the bright, coherent, polarized, energy-tunable, and monochromatic Xray beams can be used for fluorescence, scattering, diffraction, or absorption experiments. Furthermore, the recent developments made in the domain of X-ray optics ${ }^{13,14}$ offer the possibility to realize microscopy experiments with hard $\mathrm{X}$-rays not only on the micrometer but nowadays also in the sub-micrometer range. ${ }^{15}$ Indeed, at synchrotron radiation facilities, current efforts strive towards nano-focused X-ray beams ${ }^{16-21}$ with the inherent challenges on the beam position stability. At the same time focusing optics are, in combination with broadband X-ray sources, more and more commonly used in research laboratories to realize micro-XRF experiments. ${ }^{22-28}$ A permanent laboratory-based setup offers the advantages of low costs and better accessibility and, thus, a more flexible preparation and scheduling of the measurements than at external synchrotron radiation facilities.

In the present paper, the implementation of a focused $\mathrm{X}$-ray beam source in the wavelength-dispersive von Hamos spectrometer of the University of Fribourg ${ }^{29}$ to perform inhouse micro-focused XRF measurements is described. The novel X-ray source was designed so that all existing capabilities of the spectrometer are preserved. Two polycapillary halflenses are used to focus the Bremsstrahlung produced by a $50 \mathrm{~W}$ air-cooled micro-spot $\mathrm{W}$ anode $\mathrm{X}$-ray tube onto the sample. The first polycapillary half-lens is mounted directly on the X-ray tube and serves to collimate the Bremsstrahlung

\footnotetext{
a) Author to whom correspondence should be addressed. Electronic mail:
} yves.kayser@psi.ch radiation. It provides a parallel X-ray beam with a welldefined direction of propagation as well as a constant photon flux along the beam path. The second polycapillary half-lens is installed approximately $45 \mathrm{~cm}$ downstream in front of the sample in order to focus the collimated beam to a spot of about $50 \mu \mathrm{m}$. It can be noted here that as compared to the divergent beam emitted by a conventional X-ray tube the collimated micro-focus X-ray beam restricts the fluorescence production to the sample only, which results in a diminution of the background events in the spectrometer detector. In addition, as in the von Hamos setup the energy resolution depends on the size of the effective fluorescence source, a smaller instrumental broadening is obtained when using a micro-focused beam and the spectrometer can be operated in the slit-less geometry.

The laboratory-based micro-XRF setup was used to investigate Er-doped $\mathrm{SiO}_{2}$ optical fibers that are being developed at the Institute of Applied Physics (IAP) of the University of Bern using a new low-cost and time-efficient manufacturing process. The fibers fabricated with this new method, however, are characterized by quite important light losses due to scattering processes. Actually, the dopant distribution within the optical fiber is unclear and strongly affects the quality of the produced fibers. The new XRF setup was therefore used to characterize the dopant homogeneity within the core region of the fiber section to get a deeper understanding of the production process and a better control of the fiber fabrication parameters.

\section{METHODOLOGY AND INSTRUMENTATION}

The new micro-focused XRF setup is based on the highresolution von Hamos curved crystal spectrometer of the University of Fribourg. ${ }^{29}$ This reflection-type wavelengthdispersive geometry was first presented in 1933 by L. von Hamos $^{30}$ and comprises three main elements: an effective Xray source, a cylindrically curved crystal (radius of curvature 


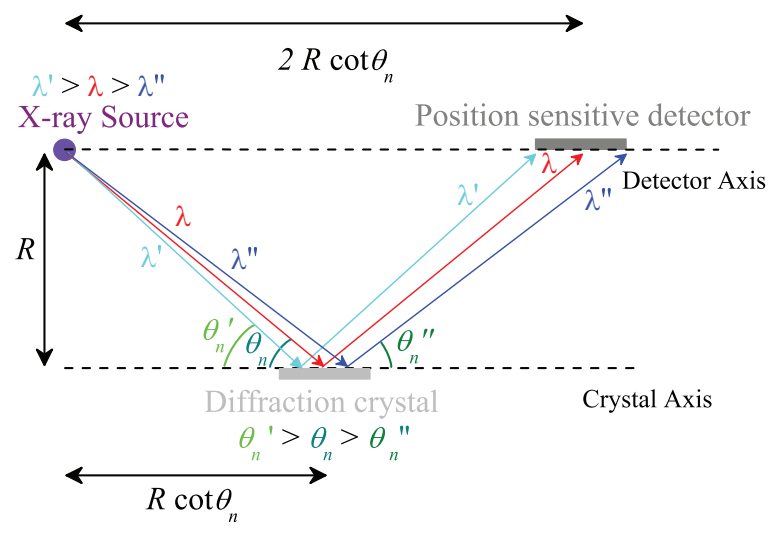

Extended

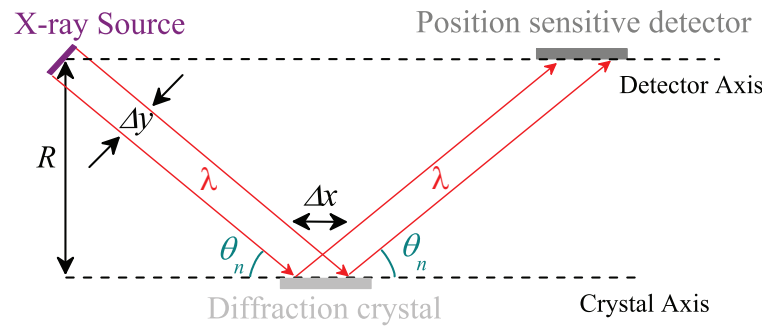

FIG. 1. Top view of the von Hamos geometry (see text for further details). The fluorescence radiation emitted from a point source is diffracted by the crystal towards the detector. The positions of the crystal and the detector are dictated by the Bragg law (Eq. (1)) while their horizontal extension allows to cover a certain range of wavelengths (upper panel). A source with a finite size in the dispersion plane of the spectrometer affects the experimental energy resolution since the Bragg condition is satisfied over an interval $\Delta x$ on the dispersion axis (lower panel)

$R$ ), and a position sensitive detector (Fig. 1, upper panel). The curvature of the crystal allows for an enhanced collection efficiency by focusing onto the detector the radiation emitted by the X-ray source in the non-dispersive direction. The source and the detector lie in the dispersion plane containing the axis of curvature of the crystal.

Among the X-ray photons emitted isotropically by the source only those which satisfy the Bragg condition ${ }^{31}$

$$
n \lambda=2 d * \sin \theta_{n}
$$

give rise to constructive interferences. In the Bragg law, $n$ stands for the diffraction order, $\theta_{n}$ for the corresponding Bragg angle, $d$ for the crystal lattice spacing constant, and $\lambda$ for the wavelength of the measured X-rays.

At a fixed position of the spectrometer elements, data are collected over an energy range of several tens to several hundreds of $\mathrm{eV}$ (Fig. 1, upper panel). This presents a considerable advantage with respect to wavelength-dispersive spectrometers based on point-by-point scanning methods and compensates partially for the lower collection efficiency in comparison to scanning-based multicrystal setups. The covered energy range depends on the Bragg angle $\theta_{n}$ and on the extension in the dispersion plane of either the crystal or, as in the case of the Fribourg spectrometer, of the detector. To cover wider energy domains, the central Bragg angle can be changed by translating both the crystal and detector along axes that are parallel to the crystal curvature axis, the source- to-crystal and crystal-to-detector distances being kept equal to $R / \sin \theta_{n}$.

The energy resolution of the spectrometer can be derived from the Bragg law. It is given by

$$
\Delta E=\cot \theta_{n} * E * \Delta \theta_{n} .
$$

As shown by the above equation the energy resolution is proportional to the angular resolution $\Delta \theta_{n}$ and improves for increasing Bragg angles. For infinitely narrow sources, the angular resolution is defined by the Darwin width of the crystal and is of the order of $\mu \mathrm{rad}$ for pure non-synthetic crystals. If, however, the source has a horizontal extension $\Delta y$ in the emission direction, the angular resolution depends mainly on the source width. Indeed, the Bragg condition is satisfied in this case over an interval $\Delta x$ which depends on the source size $\Delta y$ and the crystal position (Fig. 1, lower panel). For a finite source size, the relative energy resolution becomes

$$
\frac{\Delta E}{E}=\frac{\Delta y * \cos \theta_{n}}{R} .
$$

As it can be seen from Eq. (3), the relative energy resolution improves not only with the Bragg angle and the radius of curvature but also with a decreasing source size, justifying thus the use of micro-focused beams to produce the sample fluorescence. ${ }^{32}$ On the other hand, since the efficiency of the spectrometer diminishes with $R$, a compromise between luminosity and energy resolution has to be made for the radius of curvature. $^{29}$

Since the radiation from conventional high-power sidewindow X-ray tubes is emitted within cones with angular apertures of approximately $30^{\circ}$, large sample areas are irradiated. Thus, unless very narrow samples are used, a significant loss in the spectrometer resolution may result. On the other hand, small spot sizes on the sample are difficult to achieve with such conventional laboratory X-ray sources because a collimation of the primary X-ray radiation with pinholes implies a tremendous loss in flux. For the von Hamos spectrometer of the University of Fribourg, the spectrometer resolution was preserved by limiting the horizontal extension $\Delta y$ of the fluorescence source in the emission direction using a narrow rectangular slit having an adjustable width. ${ }^{29}$ The slit center lies on the axis of curvature of the crystal and the slit aperture in the dispersion plane determines the spectrometer resolution and luminosity (Fig. 2, left panel). In this modified von Hamos geometry, the slit can be regarded as the effective or geometrical source of radiation which corresponds to a narrow line source. The physical source, i.e., the sample area irradiated by the primary radiation, is located behind the slit with respect to the crystal view-point. Since the slit is located at a fixed position and the crystal position changes depending on the Bragg angle, the sample position should be adjustable along the primary beam direction. Note that the orientation of the slit is automatically adjusted onto the sample position by a dedicated mechanical system so that the slit width remains the same regardless of the Bragg angle (Fig. 2, left panel). With this design the von Hamos spectrometer can be operated with conventional high-power X-ray tubes. However, as the flux incident on the sample decreases quadratically with the distance $d$ separating the sample and the 

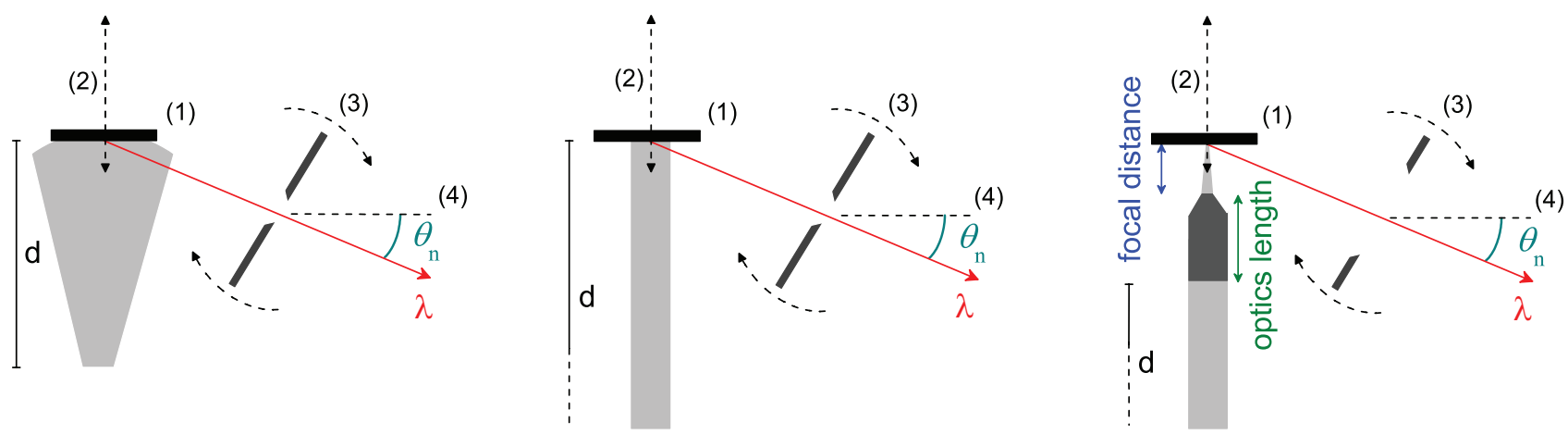

FIG. 2. Schematic illustration of the different configurations for the excitation of the photo-fluorescence signal (wavelength $\lambda$, Bragg angle $\theta_{n}$ ). The strongly divergent primary beam from a standard X-ray tube involves an incident photon flux on the sample which depends on the distance $\mathrm{d}$ separating the X-ray tube and the sample (left panel). This inconvenience is circumvented by using a micro-focused X-ray tube with a collimating polycapillary optics which delivers a quasi-parallel primary beam for the fluorescence excitation (middle panel). This allows positioning the X-ray tube at a larger distance $\mathrm{d}$ from the sample without loss in flux and, if micro-sized X-ray spots on the sample are needed, to insert a focusing polycapillary optics in front of the sample. In this case, the slit placed between the sample and the crystal is no longer needed and can be removed or widely opened (right panel). (1) Sample, (2) sample axis, (3) orientable slit, (4) detector axis, $\mathrm{d}=$ source to sample/polycapillary optics distance.

anode of the X-ray tube, the latter should be mounted as close as possible to the sample. For the spectrometer discussed in the present paper, this distance ranges from $3.4 \mathrm{~cm}$ for the smallest Bragg angle (about $24^{\circ}$ ) to $6.8 \mathrm{~cm}$ for the largest one (about $61^{\circ}$ ).

Due to this short and variable separation distance and the wide aperture of the Bremsstrahlung emission cone the implementation of a focusing X-ray optics in order to realize micro-XRF experiments with conventional X-ray sources is completely impractical. For this reason, the high-power $(3 \mathrm{~kW}) \mathrm{X}$-ray tube was replaced by a low-power $(50 \mathrm{~W})$ micro-spot X-ray tube equipped with collimating optics. The latter is mounted at the X-ray exit side of the tube and positioned accurately by the manufacturer so that its focal point coincides with the micro-sized impact point of the electrons on the X-ray tube anode. Thanks to the collimation a larger part of the Bremsstrahlung contributes to the sample fluorescence production and the photon intensity incident on the sample is independent of the distance between the X-ray tube and the sample (Fig. 2, middle panel). This allows to mount the X-ray tube at a larger distance from the sample (about $50 \mathrm{~cm}$ ). Despite the longer path of the primary X-ray beam, the absorption can be neglected since the spectrometer is operated at a pressure of typically $10^{-6}$ mbar. Furthermore, with the so-obtained gain in space and nearly parallel collimated beam, an additional optics for focusing the primary radiation onto the sample can be inserted (Fig. 2, right panel). Both the collimating and focusing optics are polycapillary half-lenses.

X-ray polycapillary optics consist of an array of about $10^{5}$ individual gently curved hollow glass capillaries through which the X-rays are guided and redirected by multiple total external reflection on the capillary walls. Since their first description $^{33,34}$ their production and design have been optimized by fusing and drawing (monolithic design) as well as tapering off the capillaries. ${ }^{35,36}$ Polycapillary optics can be either full-lenses, realizing a point-to-point focus or, like in the presented setup, half-lenses which can be either used to collimate the radiation emitted from a point source into a par- allel beam or to focus a parallel beam towards a focal point in order to increase the photon flux. ${ }^{37}$ The main parameters of a polycapillary optics are the focal spot size $\left(10^{-5} \mathrm{~m}\right)$ or acceptance angle (up to $20^{\circ}$ ), the input and/or output focal distance(s) $\left(10^{-3}-10^{-2} \mathrm{~m}\right)$, the transmission $(0.1-0.6)$, and the gain $\left(10^{2}-10^{3}\right.$ for focusing polycapillary optics). ${ }^{38}$ In general, the characteristics vary for each polycapillary optics and depend on the individual optimized design (length, diameter, and radius of curvature of the capillaries, entrance and exit apertures). ${ }^{39}$

Typical application domains for polycapillary optics are $\mu$-XRF, absorption, or diffraction experiments with the aim to realize spatially resolved measurements, to increase the detection efficiency, ${ }^{35,36,40,41}$ or to optimize the background conditions by shielding the detector from scattered particles or X-ray radiation. ${ }^{37,40}$ Arranged in the confocal geometry, ${ }^{42-48}$ 3D-measurements with micrometer resolution can also be realized. In general, the advantages offered by polycapillary optics are the compact size, the spatial acceptance of the incoming X-ray beam, and a broad usable energy range. ${ }^{48}$ The latter point, and also the achromaticity of polycapillary optics, ${ }^{40}$ make it possible to use them in combination with laboratory X-ray sources. For focusing polycapillary half-lenses, the size and position of the produced focal spot do not depend sensitively on the size and position along the optical axis of the X-ray source. ${ }^{48}$ However, the focal spot size depends slightly on the X-ray energy $40,48,49$ since the critical angle for total external reflection varies inversely with this parameter. In addition to the penetration through the capillary glass walls, this limits the transmission of high X-ray energies but allows on the other hand to use polycapillary optics as low-pass filters. The lower energy limit for the usable X-ray energies is given by the absorption at the entrance and exit windows of the optics. ${ }^{36,48}$ Most polycapillary optics are used in an energy domain of $1-40 \mathrm{keV}^{41}$

In the presented setup, the collimating polycapillary halflens was already mounted by the manufacturer on the lowpower micro-spot X-ray tube. The latter was then fixed on a 
side beam port of the spectrometer chamber via a positioning system equipped with micro-screws in order to align the collimated X-ray beam with respect to the geometry of the von Hamos spectrometer. Indeed, for ease of operation reasons, the collimated X-ray beam had to be contained in the dispersion plane of the spectrometer and perpendicular to the detector axis. The alignment of the beam along the sample positioning axis was verified with position-sensitive diodes. The focusing polycapillary half-lens was then mounted on a five-axis manipulator which was itself fixed mechanically to the sample carriage stage driving the sample support system along the primary beam direction. The alignment of the focusing polycapillary optics is thus independent of the sample position along this direction while the sample can still be scanned transversally through the focal spot. The housing containing the focusing polycapillary optics is $50 \mathrm{~mm}$ long and has a downstream aperture of $5.3 \mathrm{~mm}$ and an upstream aperture of $12 \mathrm{~mm}$, both being closed with Be windows (12.7 $\mu \mathrm{m}$ thickness). The nominal diameter of the focal spot is $50 \mu \mathrm{m}$ and the focal distance is $8 \mathrm{~mm}$. The choice of the focal output distance of $8 \mathrm{~mm}$ was dictated by spatial constraints. Indeed, the polycapillary optics output should not be on the detection path (from the sample towards the diffraction crystal) of the fluorescence X-rays and the possibility for a sample rotation was to be preserved.

The five-axis manipulator (Fig. 3), motorized for reasons of compactness and accuracy with piezo motors, allowed for three translational and two rotational motions. One translational axis is parallel to the primary beam direction while the two others are transversal to it, one being contained in the dispersion plane of the spectrometer and the other one perpendicular to it. The rotation axes coincide with the two transversal translation axes and serve to orientate the focusing optics relatively to the primary beam direction in order to maximize the transmission through the polycapillary. The angular alignment relatively to the incident beam path is cru-

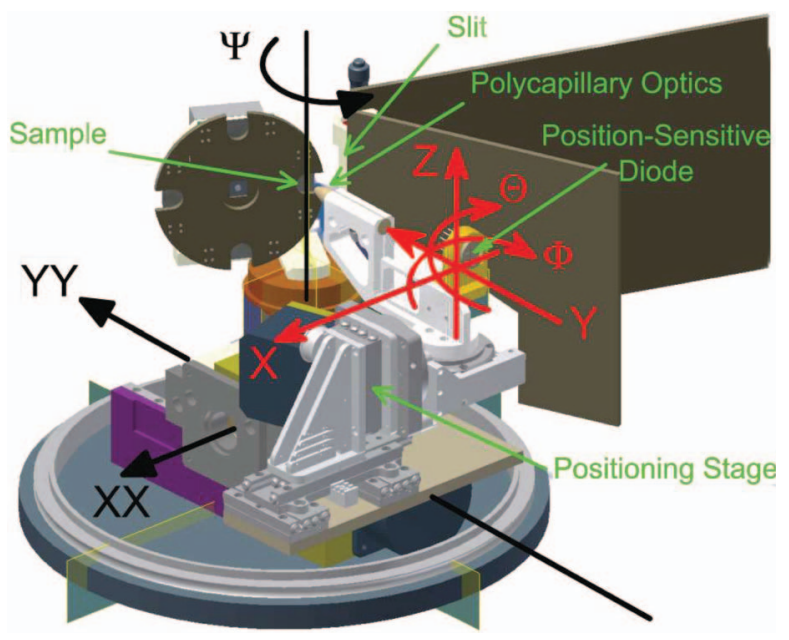

FIG. 3. Drawing of the five-axis positioning stage (red axes) used for the manipulation of the focusing polycapillary optics. The manipulator is attached to the motorized carriage permitting to move the sample along the axis YY which is parallel to the primary beam axis Y. Note that the transverse motions of the focusing optics and sample along the parallel axes $\mathrm{X}$ and $\mathrm{XX}$ are fully independent. cial since a misalignment of 2 mrad in the horizontal or vertical angular position results in flux losses of about 50\%. The two transverse translational axes are necessary to center the polycapillary optics on the direction of the collimated beam with the help of a position-sensitive diode. The latter is fixed to the polycapillary optics holder, the relative position between the center of the diode and of the optics entrance being known. The third transversal axis serves to adjust the distance between the sample surface and the exit of the focusing optics to the focal distance and, thus, to maximize the gain. This last adjustment step and the angular alignment are performed by monitoring with a video system through a viewport the focused beam on a fluorescence screen placed in the same vertical plane as the sample surface. The fluorescence screen is made of a $4 \mu \mathrm{m}$ thick $\mathrm{Gd}_{2} \mathrm{O}_{2} \mathrm{~S}$ :Tb layer deposited on a polished Al plate. It is characterized by a $2.6 \mathrm{~ms}$ decay time and an ultra fine grain size for good spatial resolution. Either the spot size or the brightness of the irradiated area is monitored.

\section{INSTRUMENTAL PERFORMANCE}

The collimated and focused primary X-ray beams were both characterized in terms of spatial extension and spectral output. First, the extension of the collimated beam and its orientation with respect to the spectrometer geometry were checked. To this end, the diode mounted on the five-axis manipulator next to the focusing polycapillary optics was scanned through the collimated beam with steps of $0.5 \mathrm{~mm}$ in the horizontal and vertical directions. The primary beam delivered by the X-ray tube and collimating polycapillary half-lens was found to be symmetric and well described by a 2D-Gaussian with a full width at half maximum of about $5.5 \pm 0.1 \mathrm{~mm}$ when taking into account the size of the diode $(7.6 \mathrm{~mm})$ (Fig. 4). The retrieved width of the collimated beam depends, however, slightly on the high voltage applied to the X-ray tube. It varies horizontally and vertically by about $0.2 \mathrm{~mm}$ with a trend towards smaller values for increasing high voltage values, i.e., increasing average energies of the emitted Bremsstrahlung. Indeed, the divergence of the polycapillary half-lens is related to the critical angle of total external reflection which depends inversely on the X-ray energy. The center position of the 2D-Gaussian, however, was

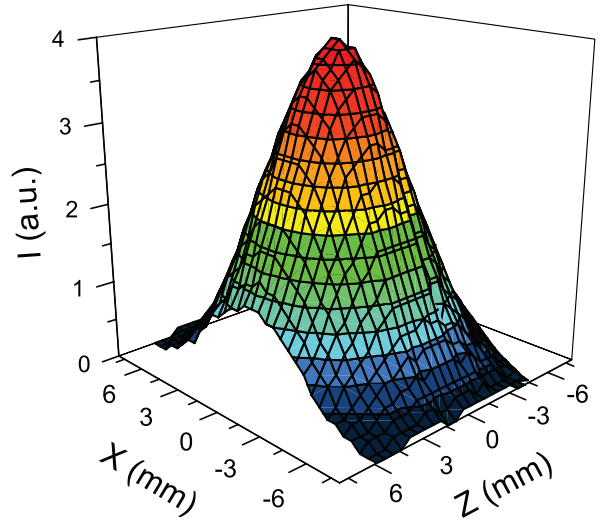

FIG. 4. Map of the intensity distribution of the collimated primary beam produced by the low-power X-ray tube in both transverse directions. 
found to be stable in both directions within $0.1 \mathrm{~mm}$. From the change in the central position along the primary beam direction it was deduced that the collimated beam was tilted by 4.6 , respectively, $5.6 \mathrm{mrad}$, in the horizontal and vertical directions. Over the travel range of the sample along the beam direction $(34 \mathrm{~mm})$ this corresponds to a total displacement of about $0.3 \mathrm{~mm}$. From the variation of the width of the 2DGaussian along the primary beam direction, a divergence of about $6 \mathrm{mrad}$ of the collimated beam in both transversal directions was deduced.

The diameter of the X-ray spot produced by the focusing polycapillary half-lens can be determined experimentally by a wire or knife edge scan, or alternatively in two dimensions with a pinhole or position-sensitive detector. For the present setup, the focal spot size, which determines the spatial resolution for mapping applications, was measured by means of the knife-edge method using a $25 \mu \mathrm{m}$ thick Sc foil (see Fig. 5, upper panel). The intensity of the $\mathrm{Sc} \mathrm{K} \alpha_{1}$ fluorescence line was monitored as the $\mathrm{Sc}$ foil was scanned transversally through the focal spot. The measurements were repeated for different dis-
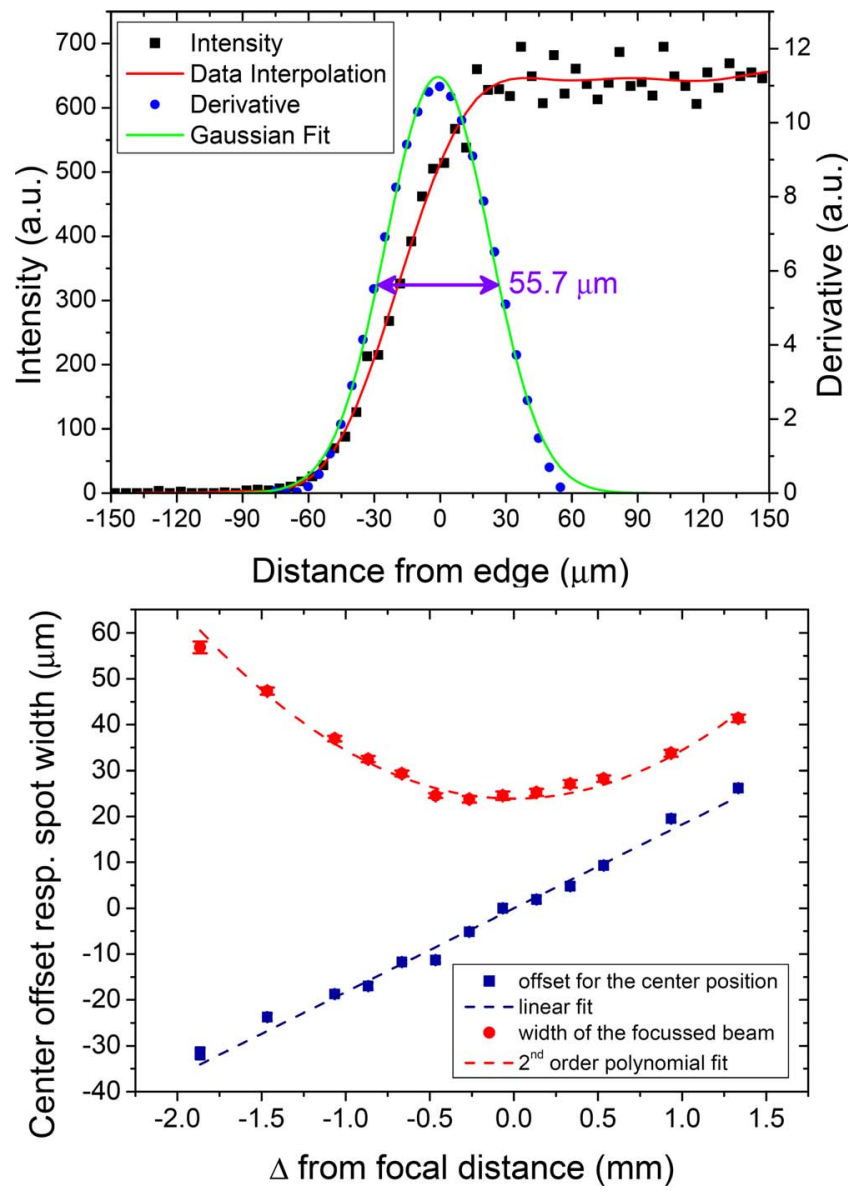

FIG. 5. Beam spot size at the focal distance of the focusing polycapillary half-lens as measured by means of a knife edge scan (see text for details). The size of the focal spot was determined from a Gaussian fit of the derivative of the intensity scan (top panel). The variation of the size and position of the beam spot when changing the distance between the polycapillary optics exit and the sample surface around the focal distance is shown in the bottom panel. The depth of focus of the optics and the required sensitivity for positioning the sample with respect to the half-lens can be estimated. The linear variation of the center position is due to the non-perfect alignment of the X-ray tube and positioning stage with respect to the spectrometer geometry. tances between the foil and the polycapillary optics exit. The $\mathrm{X}$-ray tube setting was $50 \mathrm{kV}$. The minimum spot size was found to be $55.7 \mu \mathrm{m}$ and the variation of the spot size with the separation distance between the sample and the Sc foil was well fitted by a second order polynomial (Fig. 5, lower panel). As expected, for a lower voltage applied to the X-ray tube the minimum spot size of the focused beam was found to be at the same position but larger $(\approx 20 \%$ for a voltage of $20 \mathrm{kV}$ ) since the average energy of the Bremsstrahlung contributing to the fluorescence radiation is lower. Note that for similar reasons the retrieved spot size may also depend on the element used for the knife edge scan. A systematic study with different elements would allow to partially elude this dependence. A further conclusion from these measurements is that the distance between the polycapillary optics and the sample surface can be accurately adjusted to the focal distance by surveying the size of the focused spot on the fluorescence screen, the depth of focus of the X-ray optics offering in addition a certain tolerance (increase of the spot width by $10 \mu \mathrm{m}$ for an offset of $1 \mathrm{~mm}$ from the focal distance).

The spectral distributions of both the collimated and focused beams were measured by means of a silicon drift detector (SDD) for different high voltages and a tube current of $50 \mu \mathrm{A}$ (Fig. 6). The SDD detector was installed inside the spectrometer chamber at the sample position and calibrated with an ${ }^{241} \mathrm{Am}$ radioactive source. The measurements were performed in vacuum. For the collimated beam, a $\mathrm{Pb}$ pinhole

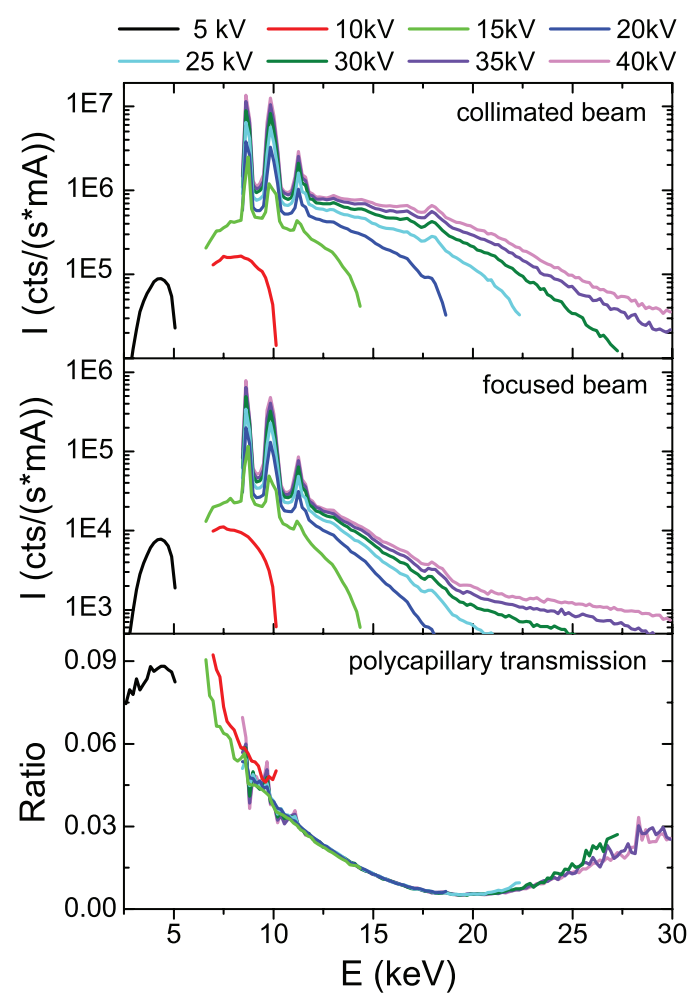

FIG. 6. Spectral distributions of the collimated (top panel) and focused (middle panel) X-ray beams for different high voltages of the X-ray tube. For each high voltage, the intensity ratio between the focused and collimated beams allows to retrieve the transmission through the focusing polycapillary half-lens (bottom panel). The lower energy limit for the usable energy range is due to the absorption by the Al foils inserted in front of the SDD. For further details see the text. 
of $0.6 \mathrm{~mm}$ in diameter which was centered on the maximum of the intensity distribution was placed in front of the detector to avoid saturation of the latter. Moreover, depending on the high voltage applied to the X-ray tube, Al filters were mounted in front of the SDD for both the collimated and focused beams $(208 \mu \mathrm{m}$ thickness at 10 and $15 \mathrm{kV}, 416 \mu \mathrm{m}$ thickness at higher voltages). From the known spatial extension of the collimated beam, the total emitted photon flux and the flux intercepted by the focusing polycapillary optics could be recalculated. The recorded spectra were corrected for the transmission through the $\mathrm{Al}$ foils and spatial acceptance of the pinhole, respectively, polycapillary entrance, as well as for the detector dead time and efficiency. For high voltages of the $\mathrm{X}$-ray tube above the $\mathrm{W} \mathrm{L}_{3}$ edge $(10.207 \mathrm{keV})$, the characteristic $\mathrm{L} X$-ray emission from the tube anode $(\mathrm{L} \alpha, \mathrm{L} \beta$, and $\mathrm{L} \gamma$ lines with energies ranging between 8.335 and $11.286 \mathrm{keV}$ ) show up on top of the produced Bremsstrahlung. From the intensity ratios of the spectra measured with the collimated and focused beams, the transmission through the focusing polycapillary as a function of the X-ray energy could be determined (Fig. 6, lower panel). In this case, only the different spatial acceptances and detector dead times have to be considered. The error was estimated to be of the order of $10 \%$. The gap from 5 to $7 \mathrm{keV}$ is due to the absorption in the $\mathrm{Al}$ foils, but the transmission value can be estimated through interpolation. The increase in the intensity ratio above $20 \mathrm{keV}$ can be explained by hard X-rays which are not reflected at the polycapillary glass walls because of a too large incidence angle. Note that the Sc knife edge scan discussed before was almost not affected by this effect because for Sc (K-edge at $4.492 \mathrm{keV}$ ) X-ray photons above $20 \mathrm{keV}$ do not significantly contribute to the production of the $\mathrm{K} \alpha$ fluorescence signal.

Furthermore, the spectrometer luminosity was compared for different fluorescence lines (corresponding absorption edges between $1.6 \mathrm{keV}$ and $10 \mathrm{keV}$ ) with three different $\mathrm{X}$-ray sources, namely, a standard high-power water-cooled Au X-ray tube (max. power $3000 \mathrm{~W}$ ), the low-power $\mathrm{W}$ anode $\mathrm{X}$-ray tube (max. power $50 \mathrm{~W}$ ) with the collimating X-ray optics, and finally the same low-power collimated X-ray source but with the focusing polycapillary half-lens inserted in front of the samples. In the last two cases, the spectrometer lumi- nosity was found to be about an order of magnitude smaller than the one corresponding to the high-power X-ray tube (for identical tube currents). With the focused beam the luminosity was comparable within a few percent to the luminosity of the setup with the collimated beam, except for fluorescence lines corresponding to absorption edges above $8 \mathrm{keV}$, i.e., absorption edges lying in the energy range where the transmission through the focusing polycapillary was found to significantly decrease (Fig. 6, lower panel). Thus, below $8 \mathrm{keV}$, losses due to the transmission through the focusing polycapillary halflens are fully compensated by the gain. On the other hand, the gain provided by the collimated beam with respect to the high-power X-ray tube (which is a strongly divergent source) is markedly attenuated by the slit placed between the sample and the crystal because the width of the latter $(0.2 \mathrm{~mm})$ is much smaller than the size of the collimated beam (about $8 \mathrm{~mm}$ ) (see Fig. 2). All spectrometer parameters like the sample, crystal, and detector position, slit width (except for the focused beam), diffraction crystal, diffraction order, and chargecoupled device (CCD) detector settings as well as the X-ray tube high voltage were identical for a given fluorescence line. The results for the Sc K $\alpha$ fluorescence line are shown in Fig. 7. However, the slightly different atomic numbers of the anode elements (about 7\% less Bremsstrahlung intensity for the $\mathrm{W}$ anode and a different mean energy of the emitted radiation) and the decreasing transmission with increasing X-ray energy through the collimating polycapillary optics were not considered. Regarding the signal-to-background ratio, the latter was found to improve in the cases of the collimated and focused beams despite a lighter shielding around the sample. Indeed, the background originating from the scattering of the primary radiation by the sample environment is small as compared to the case of the high-power X-ray tube.

The energy resolution of the spectrometer which, depending on the energy of the measured X-rays, is about 1-3 $\mathrm{eV}$ in the standard configuration was improved by up to $15 \%$ when using the setup with the collimated or the focused beam. The latter two configurations provided similar resolutions. In both cases, the improvement in resolution can be explained by the spatial distribution of the incident radiation on the sample. In the case of the collimated beam, despite the
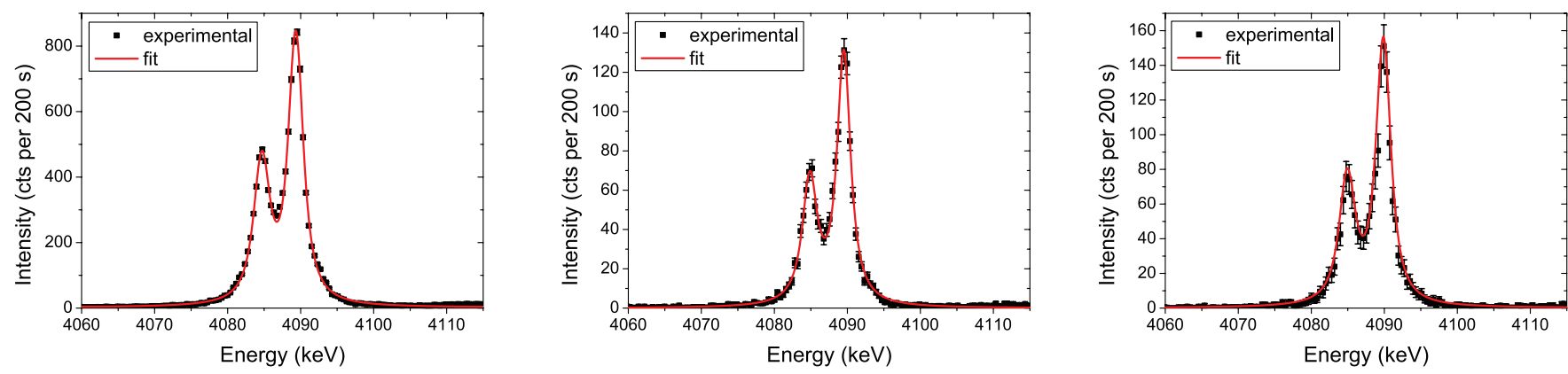

FIG. 7. Experimental spectra of the Sc K $\alpha$ fluorescence line from a $25 \mu \mathrm{m}$ thick foil using the divergent radiation beam from the high-power X-ray tube (left panel), the low power X-ray tube with collimated optics (middle panel), and the low power X-ray tube with both the collimated and focusing optics (right panel). The X-ray tubes were operated at a nominal voltage of $20 \mathrm{kV}$ and the spectra were normalized to a tube current of $10 \mathrm{~mA}$. It can be observed that with the collimated and focused beams the spectrometer luminosity is significantly lower than with the high power X-ray tube (13\% and $15 \%$, respectively), whereas a fit of the experimental spectra reveals that the background conditions are significantly improved (signal-to-background ratio better by $50 \%$ ) in the case of the low power X-ray source. The same holds for the experimental resolution (improvement from $1.22 \mathrm{eV}$ down to $1.12 \mathrm{and} 1.14 \mathrm{eV}$, respectively). 
fact that the size of the effective source of radiation is mainly determined by the width of the slit placed on the dispersion axis, the sample area contributing to the fluorescence radiation is better defined than in the case of the widespread radiation delivered by a standard X-ray tube. As mentioned before, no slit is needed when using the focused beam. However, the physical source, i.e., the irradiated sample area is not located on the dispersion axis but behind it, the distance between the physical and the geometrical source varying between 2.74 and $5.22 \mathrm{~cm}$ depending on the Bragg angle. Thus, according to the von Hamos geometry, the size of the geometrical source which is defined by the spatial extension on the dispersion axis of the fluorescence radiation accepted by the crystal is significantly wider than the physical source. Hence, the spectrometer resolution benefits only partially from the smaller size of the physical radiation source because of the modified von Hamos geometry.

\section{APPLICATION}

The first application of the described laboratory-based micro-focus wavelength-dispersive setup was the analysis of optical fibers produced by the IAP Bern. The aim was to control the homogeneity of the dopant distribution in the produced fibers by mapping the elemental distribution within transversal fiber sections.

Optical fibers are routinely used in the telecommunication domain and in laser amplifier systems. The established manufacturing method for fibers used in industrial applications is the modified chemical vapor deposition (MCVD) technique ${ }^{50}$ which allows to produce fibers of very high optical quality. On the other hand, MCDV is a very complex and consequently costly procedure. However, depending on the fiber length, the purity and the inherent signal losses may not be of prime importance. In this case, the sol-gel technique $e^{51}$ presents a manufacturing alternative in terms of handling and flexibility while the quality of the produced fibers is almost similar to those produced by means of MCVD. In addition, more complex fiber geometries with multiple cores or a dopant concentration gradient, respectively, multiple doping with different elements, can be realized.

In the same perspective, the IAP Bern has developed a new approach to dope optical fibers used in laser applications. ${ }^{52}$ Briefly, the new manufacturing method consists in filling fiber preforms with a mix of granulated silica, alumina, and oxides of the dopants and drawing afterwards the filled tubes to fibers in a standard drawing tower. The granulates can be treated by melting them with a $\mathrm{CO}_{2}$ laser in order to minimize the contamination. A milling of the vitrified pellets ensures a more homogeneous dopant mixing during the drawing of the fiber. The granulates are then filled in a quartz or Pyrex preform which may in a consecutive step be inserted coaxially in another cylindrical form. In the latter approach, the free space is filled with silica powder. The outermost part (the preform or the surrounding form) composes the cladding of the fiber. Finally, the whole tube is evacuated and preheated. Afterwards the tube is inserted in a standard drawing tower equipped with a furnace. The latter is necessary for a homogeneous annealing. The main parameters during the fiber drawing are the insertion speed from the top into the furnace, which has an influence on the final cladding diameter, and the temperature of the furnace, which allows to minimize the viscosity of silica. During the drawing of the fiber no modification of the preform geometry occurs and the cladding-tocore diameter ratio is preserved. Fibers with metallic dopants can be produced in the same manner. ${ }^{53,54}$

Like the sol-gel technique, this manufacturing approach is time and cost efficient, versatile and flexible. Unlike other methods, fibers with virtually any cladding-to-core geometry, respectively, any dopant concentration levels in the core can be produced. For broadband emission applications, like confocal microscopy or optical coherence tomography, where a small coherence length is required, fibers with alternative geometries, i.e., fibers with several cores ${ }^{55}$ or with a multi-doped core, ${ }^{56}$ can be produced. However, compared to fibers produced by standard procedures, the fibers produced by the IAP Bern are characterized by a stronger scattering, thus higher signal losses. Nevertheless, the produced fibers are suitable for active systems in which the fiber length is only of the order of several meters. The scattering originates from an inhomogeneous distribution (clustering, incomplete dissolution, or bubble inclusion in the core) of the dopants in the silica matrix.

In order to optimize the fiber production process, the homogeneity of the final dopant distribution needs to be more thoroughly investigated. Actually, by weighting the different used granulates, the average composition and the average refractive index of the core can be calculated very precisely but an incomplete dissolution of the core material during the fiber drawing leads to an inhomogeneous and fluctuating refractive index. As a consequence, scattering will occur and the signal will be damped. Thus, an exact knowledge of the local dopant distribution is of prime importance to characterize the fibers and support improvements in the production process.

The analyzed samples were sections from an Al-Er-P doped $\mathrm{SiO}_{2}$ fiber, the doping concentration of $\mathrm{Er}$ being of the order of 1 at. \%. Rare earth elements are chosen for doping optical fibers because of their weak interaction with the glass host material. The diameter of the analyzed fiber sections varied from 500 to $750 \mu \mathrm{m}$, the fiber core where the dopants are localized being smaller by a factor of $17 / 21$.

The analyzed fiber piece was quenched in the groove of a brass holder with the fiber cross-section facing the incident beam direction in order to preserve the spatial resolution offered by the focused beam. Brass was chosen because it was found to produce an acceptable background in the energy region of interest. Two-dimensional scans of transverse fiber sections were realized by moving the sample horizontally and vertically through the focused X-ray beam spot. Both movement axes were perpendicular to the incident beam direction. The $\mathrm{W}$ anode of the X-ray tube allowed for an efficient excitation of the L fluorescence lines of Er since the W L X-ray fluorescence lines are just above the L-shell absorption edges of $\mathrm{Er}$, resp. Ho and $\mathrm{Yb}$, other rare earth elements of interest for doping fibers. The positioning of the sample in the transverse directions with respect to the beam was realized with the help of the fluorescence screen. 
For the measurements, the von Hamos spectrometer was equipped with an $\operatorname{ADP}(2 d=10.642 \AA)$ crystal and the diffracted X-rays were measured with a $1340 \times 400$ pixels back-illuminated CCD detector having a spatial resolution of $20 \mu \mathrm{m}$ and a readout speed of $1 \mathrm{MHz}$. After test measurements with other crystals at different diffraction orders, namely, a $\mathrm{SiO}_{2}$ (2-23) in first order and a $\mathrm{SiO}_{2}(1-10)$ in third order, the ADP crystal used in the fourth diffraction order was found to provide the highest spectrometer luminosity for the Er L $\alpha$ fluorescence line. The integral reflectivity of the crystal was more important than the changes in the solid angle of detection due to different Bragg angles, i.e., different detector positions. In addition, the fourth diffraction order permitted to measure the $\mathrm{Si} \mathrm{K} \alpha$ and $\mathrm{Er} \mathrm{L} \alpha$ fluorescence lines at a single position of the sample, crystal, and detector. Indeed, the Bragg angles corresponding to the fourth diffraction order of the $\mathrm{Er} \mathrm{L} \alpha$ line and first diffraction order of the $\mathrm{Si} \mathrm{K} \alpha$ line are close enough to measure both fluorescence lines at a single position of the spectrometer components but sufficiently different to permit a spatial separation of the two fluorescence lines on the CCD. Moreover, the distinction between the two fluorescence signals was made easier by setting the appropriate energy discrimination on the recorded pixel intensities. ${ }^{57}$ Actually, test measurements performed with bulk $\mathrm{Si}$ and $\mathrm{Er}$ samples revealed only a weak flat background contribution from $\mathrm{Si}$ in the energy domain of the $\mathrm{Er} \mathrm{L} \alpha$ line.

The integral fluorescence intensities of the $\mathrm{Er} L \alpha$ line recorded during the horizontal line scans at different vertical positions of one of the investigated transverse fiber sections are shown in Fig. 8. Each experimental point corresponds to the measurement of 450-900 CCD images with an exposure time of $7 \mathrm{~s}$ per image. This exposure time represented the best compromise between the total acquisition time, which is the product of the number of images and the sum of the exposure time and detector readout time, and the electronic noise of the $\mathrm{CCD}$ detector for X-ray energies in the vicinity of the $\mathrm{Si}$ $\mathrm{K} \alpha$ line. Despite the long overall measurement time, no significant drift in the experimental setup could be observed. As shown in Fig. 8 significant fluorescence intensity fluctuations are observed, indicating a quite inhomogeneous dopant distribution. A comparison between the scan profiles of the $\mathrm{Si} \alpha \alpha$

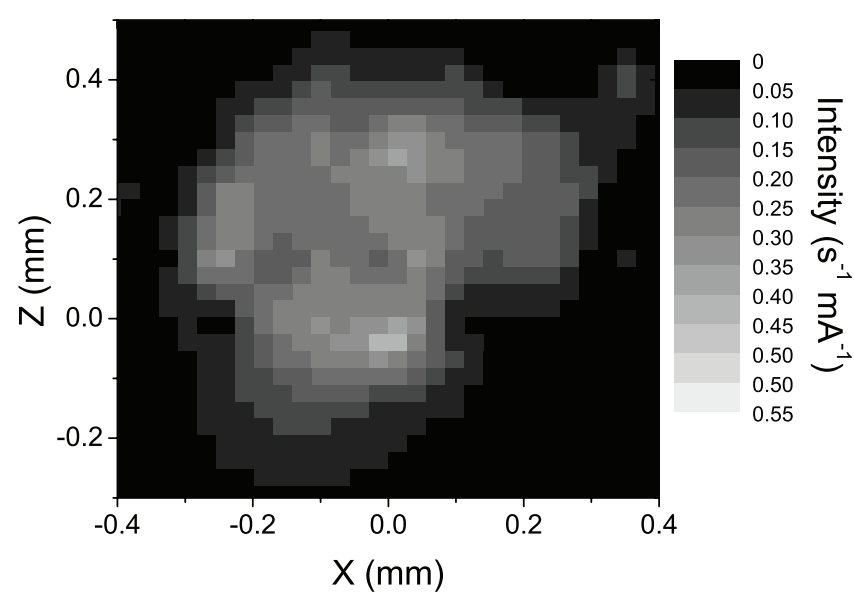

FIG. 8. Example of a two-dimensional scan with the focused beam of the Er $\mathrm{L} \alpha$ intensity emitted by a section of the analyzed fiber. and $\mathrm{Er} L \alpha$ intensity distributions permits to exclude an important dopant diffusion into the cladding. The ratio of 17/21 between the doped region and the total diameter of the fiber, which is expected to be independent from the diameter of the drawn fiber, can be indeed retrieved from the widths of the two profiles. Identical conclusions could be drawn for other analyzed fiber sections.

\section{CONCLUSION}

In summary, the implementation of a laboratory-based micro-focusing X-ray source into an existing crystal spectrometer chamber was described. The new setup permits to realize at the same time measurements with a high spatial resolution, which is important for mapping applications and localized studies, and a high energy resolution, which offers the possibility for chemical speciation when measuring X-ray fluorescence lines corresponding to electronic transitions from the valence shell.

The primary beam used to excite the fluorescence radiation was delivered by an air-cooled, low-power $50 \mathrm{kV}, 1 \mathrm{~mA}$ micro-spot X-ray tube with a collimating polycapillary halflens installed at the exit window of the tube. Thanks to the distance-independent flux of the collimated beam, it was possible to position the X-ray tube at a large distance from the sample and to insert a focusing polycapillary X-ray optics in front of the sample without modifying the spectrometer chamber. Actually, due to the limited space inside the spectrometer chamber and the polychromatic primary beam, the combination of a collimating and a focusing polycapillary half-lens represented the best and single realistic setup for the production of a micro-sized X-ray beam on the sample. The compact size and relatively easy manipulation of the polycapillary optics are further advantages of the chosen setup. Both the collimated and focused beams were characterized in terms of spatial distribution and spectral output.

The new laboratory-based micro-focusing X-ray source allows for a spatially resolved and non-destructive analysis of small samples. The new setup was therefore used to investigate different sections of tiny optical fibers manufactured at the IAP Bern by means of a new fabrication process. The dopant distribution within the core of the optical fibers was of particular interest. The present paper shows that a systematic study of the produced fibers is achievable. Indeed, the first objective which was to perform lateral elemental mapping of the concentration levels of the dopant and core atoms could be achieved. Moreover, thanks to the quite deep penetration of X-rays, it can be envisaged to study the fibers along the longitudinal direction or to analyze the pellets used in the production process. Together with a reference standard, a quantitative analysis would also be possible. ${ }^{58}$ A more systematic study aiming at elucidating the influence of the production parameters on the distribution of the fiber dopants can be foreseen together with other complementary methods, e.g., electron microscopy. The effectiveness of several measures taken in the manufacturing preparation to improve the homogeneity of the dopants distribution in the silica host matrix could be thus evaluated. 


\section{ACKNOWLEDGMENTS}

The financial support of the LiMat project and the Swiss National Science Foundation (SNF) is acknowledged. The authors would also like to recognize the assistance of the mechanicians' and electronicians' staff of the Physics Department of the University of Fribourg.

${ }^{1}$ F. Adams, B. Vekemans, G. Silversmit, B. Samber, and L. Vincze, in Handbook of Nuclear Chemistry, edited by A. Vértes, S. Nagy, Z. Klencsàr, R. G. Lovas, and F. Rösch (Springer US, 2011), pp. 1737-1759.

${ }^{2}$ S. Majumdar, J. R. Peralta-Videa, H. Castillo-Michel, J. Hong, C. M. Rico, and J. L. Gardea-Torresdey, Anal. Chim. Acta 755, 1 (2012).

${ }^{3}$ U. E. A. Fittschen and G. Falkenberg, Spectrochim. Acta B 66, 567 (2011).

${ }^{4}$ S. Frisia, A. Borsato, and J. Susini, J. Volcanol. Geotherm. Res. 177, 96 (2008).

${ }^{5}$ M. A. Denecke, A. Somogyi, K. Janssens, R. Simon, K. Dardenne, and U. Noseck, Microsc. Microanal. 13, 165 (2007).

${ }^{6}$ A. V. Chadwick, A. Berko, E. J. Schofield, A. M. Jones, J. F. W. Mosselmans, and A. D. Smith, Int. J. Archit. Herit. 6, 228 (2012).

${ }^{7}$ L. Bertrand, M. Cotte, M. Stampanoni, M. Thoury, F. Marone, and S. Schöder, Phys. Rep. 519, 51 (2012).

${ }^{8}$ S. Bohic, M. Cotte, M. Salomé, B. Fayard, M. Kuehbacher, P. Cloetens, G. Martinez-Criado, R. Tucoulou, and J. Susini, J. Struct. Biol. 177, 248 (2012).

${ }^{9}$ M. Farquharson, K. Geraki, G. Falkenberg, R. Leek, and A. Harris, Appl. Radiat. Isotopes 65, 183 (2007).

${ }^{10}$ C. G. Schroer and J.-D. Grunwaldt, Synchrotron Radiat. News 22, 23 (2009).

${ }^{11}$ M. Vespa, E. Wieland, R. Dähn, D. Grolimund, and A. Scheidegger, Cement Concrete Res. 37, 1473 (2007).

${ }^{12}$ I. M. Kempson, K. P. Kirkbride, W. M. Skinner, and J. Coumbaros, Talanta 67, 286 (2005)

${ }^{13}$ A. Guilherme, G. Buzanich, and M. Carvalho, Spectrochim. Acta B 77, 1 (2012).

${ }^{14}$ A. Snigirev, Frontiers in Optics (Optical Society of America, 2009), p. FThG2.

${ }^{15}$ G. E. Ice, J. D. Budai, and J. W. L. Pang, Science 334, 1234 (2011).

${ }^{16}$ R. P. Winarski, M. V. Holt, V. Rose, P. Fuesz, D. Carbaugh, C. Benson, D. Shu, D. Kline, G. B. Stephenson, I. McNulty, and J. Maser, J. Synchrotron Radiat. 19, 1056 (2012).

${ }^{17}$ G. Martínez-Criado, R. Tucoulou, P. Cloetens, P. Bleuet, S. Bohic, J. Cauzid, I. Kieffer, E. Kosior, S. Labouré, S. Petitgirard, A. Rack, J. A. Sans, J. Segura-Ruiz, H. Suhonen, J. Susini, and J. Villanova, J. Synchrotron Radiat. 19, 10 (2012)

${ }^{18}$ A. Buffet, A. Rothkirch, R. Döhrmann, V. Körstgens, M. M. Abul Kashem, J. Perlich, G. Herzog, M. Schwartzkopf, R. Gehrke, P. Müller-Buschbaum, and S. V. Roth, J. Synchrotron Radiat. 19, 647 (2012).

${ }^{19}$ J. Ablett, C. Kao, R. Reeder, Y. Tang, and A. Lanzirotti, Nucl. Instrum. Methods Phys. Res. A 562, 487 (2006).

${ }^{20}$ Y. Suzuki, N. Watanabe, S. Aoki, and Y. Kagoshima, Synchrotron Radiat. News 16, 44 (2003).

${ }^{21}$ J. Susini, M. Salomé, B. Fayard, R. Ortega, and B. Kaulich, Surf. Rev. Lett. 09, 203 (2002)

${ }^{22}$ S. Smolek, C. Streli, N. Zoeger, and P. Wobrauschek, Rev. Sci. Instrum. 81 , 053707 (2010)

${ }^{23}$ J. Zieba-Palus, R. Borusiewicz, and M. Kunicki, Forensic Sci. Int. 175, 1 (2008).
${ }^{24}$ A. Longoni, C. Fiorini, C. Guazzoni, S. Buzzetti, M. Bellini, L. Strüder, P. Lechner, A. Bjeoumikhov, and J. Kemmer, X-Ray Spectrom. 34, 439 (2005).

${ }^{25} \mathrm{~K}$. Tsuji, T. Emoto, Y. Matsuoka, Y. Miyatake, T. Nagamura, and X. Ding, Powder Diffr. 20, 137 (2005).

${ }^{26}$ H. Bronk, S. Röhrs, A. Bjeoumikhov, N. Langhoff, J. Schmalz, R. Wedell, H.-E. Gorny, A. Herold, and U. Waldschläger, Fresen. J. Anal. Chem. 371, 307 (2001).

${ }^{27}$ S. Bichlmeier, K. Janssens, J. Heckel, D. Gibson, P. Hoffmann, and H. M. Ortner, X-Ray Spectrom. 30, 8 (2001)

${ }^{28}$ K. Janssens, B. Vekemans, L. Vincze, F. Adams, and A. Rindby, Spectrochim. Acta B 51, 1661 (1996).

${ }^{29}$ J. Hoszowska, J.-Cl. Dousse, J. Kern, and C. Rhême, Nucl. Instrum. Methods Phys. Res. A 376, 129 (1996).

${ }^{30}$ L. von Hámos, Ann. Phys. (Leipzig) 409, 716 (1933).

${ }^{31}$ W. H. Bragg and W. L. Bragg, Proc. R. Soc. London, Ser. A 88, 428 (1913).

${ }^{32}$ J. Szlachetko, M. Nachtegaal, E. de Boni, M. Willimann, O. Safonova, J. Sa, G. Smolentsev, M. Szlachetko, J. A. van Bokhoven, J.-C. Dousse, J. Hoszowska, Y. Kayser, P. Jagodzinski, A. Bergamaschi, B. Schmitt, C. David, and A. Lücke, Rev. Sci. Instrum. 83, 103105 (2012).

${ }^{33}$ M. A. Kumakhov, Nucl. Instrum. Methods Phys. Res. A 48, 283 (1990).

${ }^{34}$ M. A. Kumakhov and F. F. Komarov, Phys. Rep. 191, 289 (1990).

${ }^{35}$ D. H. Bilderback, X-Ray Spectrom. 32, 195 (2003).

${ }^{36}$ P. J. Schields, D. M. Gibson, W. M. Gibson, N. Gao, H. Huang, and I. Y. Ponomarev, Powder Diffr. 17, 70 (2002).

${ }^{37}$ C. A. MacDonald, J. X-Ray Sci. Technol. 6, 32 (1996).

${ }^{38}$ C. A. MacDonald, X-Ray Opt. Instrum. 2010, 867049 (2010).

${ }^{39}$ M. Haschke and M. Haller, X-Ray Spectrom. 32, 239 (2003).

${ }^{40}$ T. Wolff, I. Mantouvalou, W. Malzer, J. Nissen, D. Berger, I. Zizak, D. Sokaras, A. Karydas, N. Grlj, P. Pelicon, R. Schütz, M. Žitnik, and B. Kanngießer, J. Anal. At. Spectrom. 24, 669 (2009).

${ }^{41}$ Y. Yan and W. M. Gibson, Adv. X-Ray Anal. 45, 298 (2002), available online at http://www.icdd.com/resources/axa/vol45/V45_47.pdf.

${ }^{42}$ S. Smolek, B. Pemmer, M. Folser, C. Streli, and P. Wobrauschek, Rev. Sci. Instrum. 83, 083703 (2012).

${ }^{43}$ B. Kanngießer, W. Malzer, I. Mantouvalou, D. Sokaras, and A. Karydas, Appl. Phys. A 106, 325 (2012).

${ }^{44}$ K. Nakano, C. Nishi, K. Otsuki, Y. Nishiwaki, and K. Tsuji, Anal. Chem. 83, 3477 (2011)

${ }^{45}$ I. Mantouvalou, K. Lange, T. Wolff, D. Grötzsch, L. Lühl, M. Haschke, O. Hahn, and B. Kanngießer, J. Anal. At. Spectrom. 25, 554 (2010).

${ }^{46}$ R. D. Perez, H. J. Sanchez, C. A. Perez, and M. Rubio, Radiat. Phys. Chem. 79, 195 (2010)

${ }^{47}$ K. Tsuji and K. Nakano, X-Ray Spectrom. 36, 145 (2007).

${ }^{48}$ L. Vincze, F. Wei, K. Proost, B. Vekemans, K. Janssens, Y. He, Y. Yan, and G. Falkenberg, J. Anal. At. Spectrom. 17, 177 (2002).

${ }^{49}$ A. Bjeoumikhov, N. Langhoff, S. Bjeoumikhova, and R. Wedell, Rev. Sci. Instrum. 76, 063115 (2005).

${ }^{50}$ D. Jablonowski, J. Lightwave Technol. 4, 1016 (1986).

${ }^{51}$ M. Locher, V. Romano, and H. P. Weber, Opt. Lasers Eng. 43, 341 (2005).

${ }^{52}$ R. Renner-Erny, L. D. Labio, and W. Lüthy, Opt. Mater. 29, 919 (2007).

${ }^{53}$ M. Neff, V. Romano, and W. Lüthy, Opt. Mater. 31, 247 (2008).

${ }^{54}$ M. Neff, V. Romano, and W. Lüthy, Opt. Mater. 33, 1 (2010).

${ }^{55}$ L. Di Labio, W. Lüthy, V. Romano, F. Sandoz, and T. Feurer, Appl. Opt. 47, 1581 (2008)

${ }^{56}$ L. Di Labio, W. Lüthy, V. Romano, F. Sandoz, and T. Feurer, Opt. Lett. 33, 1050 (2008).

${ }^{57}$ J. Szlachetko, J.-Cl. Dousse, J. Hoszowska, M. Berset, W. Cao, M. Szlachetko, and M. Kavčič, Rev. Sci. Instrum. 78, 093102 (2007).

${ }^{58}$ B. Kanngießer, Spectrochim. Acta B 58, 609 (2003). 